\title{
A more precise rounding algorithm for rational numbers
}

\section{Thill}

Published online: 27 August 2008

(C) Springer-Verlag 2008

\section{Erratum to: Computing (2008) 82:189-198 DOI 10.1007/s00607-008-0006-7}

The purpose of this note is to correct a mistake in the article [1]. An error crept into algorithm 4, the more precise rounding. The algorithm does not work for inputs $<1$, in that it then always gives the result $1 / 0$. The error consists in the extra condition " $X_{1} \geq X$ and" in the test following the comment "\{now look ahead: $\} "$. This extra condition is to be deleted. The correct algorithm goes as follows:

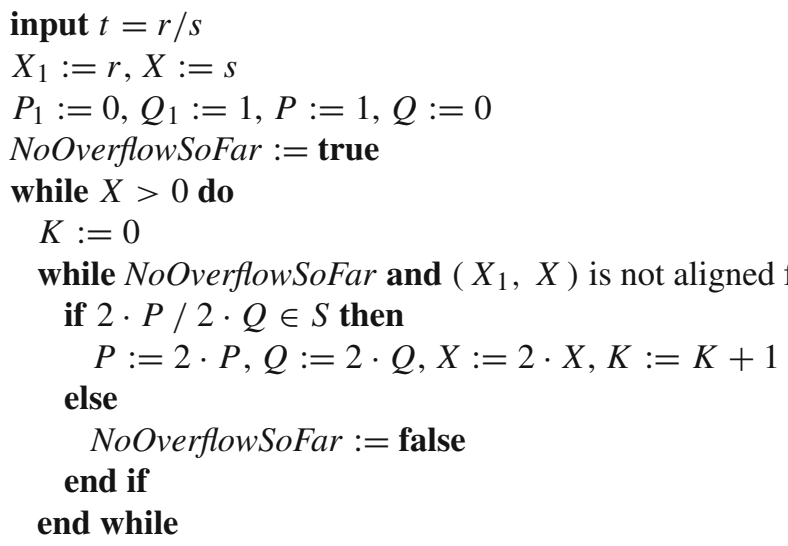

The online version of the original article can be found under doi:10.1007/s00607-008-0006-7.

M. Thill ( $\varangle)$

bd G.-D. Charlotte 53, L-1331 Luxembourg City, Luxembourg

e-mail: math@pt.lu

URL: http://web.me.com/marco_thill/ 
\{now look ahead:

if NoOverflowSoFar and $P_{1}+P / Q_{1}+Q \in S$ then

repeat

if $X_{1} \geq X$ then

NoOverflowSoFar $:=\left(P_{1}+P / Q_{1}+Q \in S\right)$

\{please note above the difference to algorithm 3 \}

if NoOverflowSoFar then

$$
P_{1}:=P_{1}+P, Q_{1}:=Q_{1}+Q, X_{1}:=X_{1}-X
$$

end if

end if

if $K>0$ then

$X_{1}:=2 \cdot X_{1}, P:=P / 2, Q:=Q / 2$

end if

$K:=K-1$

until $K<0$

else

\{recover convergent: $\}$

while $K>0$ do

$P:=P / 2, Q:=Q / 2, K:=K-1$

end while

$X:=0\{$ to exit "while" $\}$

end if

interchange $\left(X, X_{1}\right)$, interchange $\left(P, P_{1}\right)$, interchange $\left(Q, Q_{1}\right)$

end while

Result $:=\mathrm{P} / \mathrm{Q}$, Preceding Convergent $:=P_{1} / Q_{1}$

return Result, Preceding Convergent

\section{Reference}

1. Thill M (2008) A more precise rounding algorithm for natural numbers. Computing 82(2-3):189-198 